\title{
Epistemic Conditions for Rationalizability ${ }^{*}$
}

\author{
by \\ Eduardo Zambrano* \\ University of Notre Dame \\ First version: December, 1999 \\ This version: August, 2005
}

\footnotetext{
*I would like to thank session participants at the 2001 Winter Meeting of the Econometric Society, the 2000 World Congress of the Game Theory Society, the Spring 2000 Midwest Economic Theory Conference and at seminars at the University of California at Irvine and the University of Notre Dame, and especially two anonymous referees for their valuable comments on this project. All remaining shortcomings are mine.

${ }^{*}$ Department of Finance, Mendoza College of Business, University of Notre Dame, South Bend, IN 46556. Phone: (574) 631-4597. Fax: (574) 631-5255. E-mail: ezambran@nd.edu.
} 


\begin{abstract}
In this paper I show that, just as with Nash Equilibrium, there are sparse conditions, not involving common knowledge of rationality, that lead to (correlated) rationalizability. The basic observation is that, if the actual world belongs to a set of states where the set $Z$ of action profiles is played, each player knows her own payoff, everyone is rational and it is mutual knowledge that the action profiles played are in $Z$, then the actions played at the actual world are rationalizable actions. Alternatively, if at the actual world the support of the conjecture of player $i$ is $D^{i}$, there is mutual knowledge of: (i) the game being played, (ii) that the players are rational, and (iii) that for every $i$ the support of the conjecture of player $i$ is contained in $D^{i}$, then every strategy in the support of the conjectures is rationalizable. The results do not require common knowledge of anything, are valid for games with any number of players, and extend to refinements of rationalizability such as independent rationalizability and rationalizable conjectural equilibrium.
\end{abstract}

Keywords: Rationalizability, common knowledge, interactive epistemology. 


\section{Introduction}

Ever since its inception, rationalizability has been a concept intimately linked to the idea of the rationality of the players being commonly known. This intuition has been captured in Bernheim (1984, 1986), Pearce (1984), Brandenburger and Dekel (1987), and Tan and Werlang (1988). In this paper I show that, just as with Nash Equilibrium, there are sparse conditions, not involving common knowledge of rationality, that lead to (correlated) rationalizability. Indeed, the results do not require common knowledge of anything, and are valid for games with any number of players.

The stage is set by the following Preliminary Observation for Rationalizability: Fix a game g, let $Z$ be a (Cartesian) set of action profiles and let $\widetilde{S}$ be a set of states where only-and-all the profiles in $Z$ are played. If at every state in $\widetilde{S}$ the actual game is $g$, everyone is rational and it is mutually known that an action profile in $Z$ is played, then the actual action profile at each state in $\widetilde{S}$ is rationalizable for $g$.

Indeed, since every action in $Z_{i}$ arises at some state in $\widetilde{S}$, and since the action chosen at each state is in $Z_{i}$, then each action in $Z_{i}$ is a best response to beliefs whose support is contained in $Z_{-i}$; so by definition, the actions chosen at any state in $\widetilde{S}$ are rationalizable actions. ${ }^{1}$

The reader will note that this observation is analogous to the Preliminary $\mathrm{Ob}$ -

\footnotetext{
${ }^{1}$ For a formal statement of this result, together with full proof, see Section 4.
} 
servation [for Nash Equilibrium] identified in Aumann and Brandenburger (1995). ${ }^{2}$ I exploit greatly the fact that this analogy exists, and present my results closely following the style of Aumann and Brandenburger's seminal work.

Though simple, the Preliminary Observation for Rationalizability is not without interest. Note that it calls for mutual knowledge of the set of choices, with no need for any higher order knowledge. In particular, it does not call for common knowledge. For rationality and for the payoff functions, not even mutual knowledge is needed; only that the players are in fact rational, and that each knows his own payoff function.

In recent years a view has emerged that advocates interpreting solution concepts as eminently imposing restrictions on beliefs, rather than on the actions actually played. ${ }^{3}$ This is the context of my second result, which provides sufficient conditions for a product of supports of conjectures to constitute a set of profiles of rationalizable actions.

It is again a result akin to theorem in Aumann and Brandenburger (1995) (Theorem A) that which leads to rationalizability of beliefs. And, again, common knowledge plays no role. Here the conjecture of each is a probability distribution on the other's actions. One then has the following theorem: Suppose that at the current state of

\footnotetext{
${ }^{2}$ Indeed, if the collection $S$ contains only one state, the strategies chosen at $S$ constitute a Nash equilibrium and the two Preliminary Observations coincide.

${ }^{3}$ See Harsanyi (1973), Armbruster and Boege (1979), Aumann (1987), Tan and Werlang (1988), Brandenburger and Dekel (1989) and Nyarko (1998) among others.
} 
the world the support of the conjecture of every player $i$ is $D^{i}$. Suppose that at that state there is mutual knowledge of: (i) the game being played, (ii) that the players are rational, and (iii) that for every $i$ the support of player $i$ 's conjecture is contained in $D^{i}$. Then the actions that arise with positive probability under the player's conjectures are rationalizable action profiles. This is Theorem $A B$ in the paper.

It is worth noting here that this result holds for games with any finite number of players. This is in contraposition to Theorem A in Aumann and Brandenburger (1995) which holds only for two-player games. They handle the $n$-player case in their Theorem B, but to do this they require a common prior and common knowledge of conjectures, in addition to mutual knowledge of payoffs and of rationality. Those extra assumptions are not required in the present work.

When one advocates the interpretation of equilibrium as equilibrium in beliefs one is compelled to seek for the implications over observed play that follow from the beliefs being in equilibrium. ${ }^{4}$ With this in mind I present, as a corollary to Theorem $\mathrm{AB}$, a result that states that under the same hypothesis of Theorem $\mathrm{AB}$ not only the supports of conjectures are rationalizable at a state, but the actions chosen at that state are rationalizable as well.

The rest of this paper is aimed at presenting a coherent formulation and proof of the claims made above. In Section 2 I present the formal statement of the results and

\footnotetext{
${ }^{4}$ This view is prominently held by Jordan (1996) and Nyarko (1994).
} 
their proofs. In Section 3 I discuss the results.

\section{The Results}

In what follows I follow closely the standard notation and definitions as presented in Aumann and Brandenburger (1995). ${ }^{5}$ Let a game form $\mathcal{G}$ be given, that is, a finite set $N$ of players, together with a finite set of actions $A_{i}$ for each player $i$. Set $A:=\times_{i \in N} A_{i}$. A model of $\mathcal{G}$ is defined to consist of

1. for each player $i$, a set $S_{i}$ of types

and for each type $s_{i}$ of $i$

2. a probability measure on the set $S^{-i}:=\times_{j \neq i} S_{j}$ of $(n-1)$-tuples of types of the other players, where $S^{-i}$ is endowed with the product structure ( $s_{i}{ }^{\prime} s$ theory),

3. an action $\mathbf{a}_{i}\left(s_{i}\right)$ of $i$, and

4. a function $\mathbf{g}_{i}\left(s_{i}\right): A \rightarrow \Re$,

Set $S:=\times_{i \in N} S_{i}$, and call the members $s=\left(s_{1}, \ldots, s_{n}\right)$ of $S$ states. The set $S$ is also endowed with the product structure. An event is a measurable subset $E$ of $S$. Define an extension $p\left(\cdot ; s_{i}\right)$ on $S$ as follows: if $E$ is an event, define $p\left(E ; s_{i}\right)$ to be the probability that $s_{i}$ 's theory assigns to $\left\{s^{-i} \in S^{-i}:\left(s_{i}, s^{-i}\right) \in E\right\}$. For all $i$, functions

\footnotetext{
${ }^{5}$ For excellent surveys of the literature see Dekel and Gul (1997), Geanakoplos (1993); and Batigalli and Bonanno (1999).
} 
$p\left(\cdot ; s_{i}\right), \mathbf{a}_{i}\left(s_{i}\right)$ and $\mathbf{g}_{i}\left(s_{i}\right)$ are all assumed to be measurable as a function of the type $s_{i}$. I also assume that player type $s_{i}$ assigns probability 1 to $i$ being of type $s_{i}$. For a discussion on these assumptions, check the excellent discussion section in Aumann and Brandenburger (1995).

The interpretation of the model described above is that the type $s_{i}$ of player $i$ chooses action $\mathbf{a}_{i}\left(s_{i}\right)$, has a payoff function of $\mathbf{g}_{i}\left(s_{i}\right)$, and has probabilistic beliefs on $S$ at state $s=\left(s_{1}, \ldots, s_{n}\right)$ given by $p\left(\cdot ; s_{i}\right)$. I write $\mathbf{a}(s)$ for $\left(\mathbf{a}_{1}\left(s_{1}\right), \ldots, \mathbf{a}_{n}\left(s_{n}\right)\right)$, $\mathbf{g}(s)$ for $\left(\mathbf{g}_{1}\left(s_{1}\right), \ldots, \mathbf{g}_{n}\left(s_{n}\right)\right)$ and call $\mathbf{g}(s)$ the game played at $s$. Given a game $g$, a model of $g$ is simply a model of the game form associated with $g$.

Set $A^{-i}:=\times_{j \neq i} A_{j}$. A conjecture $\phi^{i}$ of $i$ is a probability distribution on $A_{-i}$. The conjecture $\phi^{i}(s)$ of $i$ at $s$ is given by the marginal of $p\left(\cdot ; s_{i}\right)$ on $A_{-i}$. I write $\phi$ for $\left(\phi_{1}, \phi_{2}, \ldots, \phi_{n}\right)$.

Player $i$ is called rational at $s$ if his action at $s$ maximizes the expectation of his payoff at $s$ when the other player's actions are distributed according to his conjecture at $s$.

If $\mathbf{x}$ is a function on $S$ and $X$ is a subset of its range, then $[\mathbf{x} \in X]$ denotes the event $\{s: \mathbf{x}(s) \in X\}$. If $\widetilde{S}$ is a subset of $S$ then $A(\widetilde{S})$ denotes the action profiles chosen at $\widetilde{S}$, that is, $A(\widetilde{S})=\{a \in A: \mathbf{a}(s)=a$ for some $s$ in $\widetilde{S}\}$. If $E$ is an event define the operator $K_{i}$ on events by

$$
K_{i}(E)=\left\{s: p\left(E ; s_{i}\right)=1\right\} .
$$


As in Aumann and Brandenburger (1995), knowledge is identified with probability one belief, so $K_{i}(E)$ is the event that player $i$ knows $E$. Set $K(E)=\cap_{i \in N} K_{i}(E)$.

I will denote a point mass probability distribution on $s$ by $1_{s}$ and a probability distribution $q$ on a set $\left\{s^{1}, \ldots, s^{n}\right\}$ by $q_{1} s^{1}+\cdots+q_{n} s^{n}$.

The version of rationalizability that I work with in this paper allows each player to believe that his opponents's actions are correlated.

A set $\times_{i \in N} Z_{i} \subseteq A$ of action profiles has the best response property if, for each player $i \in N$, every action $a_{i} \in Z_{i}$ is a best response to a belief $q_{i}$ of player $i$ whose support is a subset of $Z_{-i}$.

An action $a_{i} \in A_{i}$ is rationalizable if there is a set $\times_{i \in N} Z_{i}$ with the best response property such that $a_{i} \in Z_{i}$. The set of profiles of rationalizable actions is, therefore, the union over all the sets of action profiles that have the best response property.

\subsection{An Illustration}

Consider a model in which all types of each player $i$ have the same payoff function $g_{i}$ as depicted in Figure 2.1. Thus the game being played is known at every state. The information structure of the model is depicted in Figure 2.2. Each row denotes a state, and contains the actions chosen by the players at that state, as well as the beliefs held by the players. For example, at state $s^{1}$ Ann chooses $C$, her beliefs are given by $\frac{1}{2} s^{1}+\frac{1}{2} s^{2}$; whereas Bob chooses $c$ and his beliefs are given by $\frac{1}{3} s^{1}+\frac{1}{3} s^{4}+\frac{1}{3} s^{7}$. 
Notice that the actions chosen at $s^{1}$ are best responses to the players conjectures at $s^{1}$, that this profile belongs to the set $\{C, D\} \times\{c, d\}$, and that the support of the player's conjectures at $s^{1}$ are precisely $\{c, d\}$ and $\{C, D\}$. Hence, by definition, the actions played at $s^{1}$ are rationalizable actions.

\begin{tabular}{|c|c|c|c|}
\hline & & Bob & \\
\hline & $c$ & $d$ & $e$ \\
\hline Ann $C$ & 3,3 & 0,0 & 1,1 \\
\hline$D$ & 0,0 & 1,1 & $0,-1$ \\
\hline
\end{tabular}

Figure 2.1 


\begin{tabular}{|c|c|c|c|c|}
\hline & Ann's Action & Ann's Belief & Bob's Action & Bob's Belief \\
\hline$s^{1}$ & $C$ & $\frac{1}{2} s^{1}+\frac{1}{2} s^{2}$ & $c$ & $\frac{1}{3} s^{1}+\frac{1}{3} s^{4}+\frac{1}{3} s^{7}$ \\
\hline$s^{2}$ & $C$ & $\frac{1}{2} s^{1}+\frac{1}{2} s^{2}$ & $d$ & $\frac{1}{4} s^{2}+\frac{3}{4} s^{5}$ \\
\hline$s^{3}$ & $C$ & $1_{s^{3}}$ & $e$ & $1_{s^{3}}$ \\
\hline$s^{4}$ & $D$ & $\frac{1}{4} s^{4}+\frac{3}{4} s^{5}$ & $c$ & $\frac{1}{3} s^{1}+\frac{1}{3} s^{4}+\frac{1}{3} s^{7}$ \\
\hline States $s^{5}$ & $D$ & $\frac{1}{4} s^{4}+\frac{3}{4} s^{5}$ & $d$ & $\frac{1}{4} s^{2}+\frac{3}{4} s^{5}$ \\
\hline$s^{6}$ & $D$ & $1_{s^{6}}$ & $e$ & $1_{s^{6}}$ \\
\hline$s^{7}$ & $D$ & $\frac{1}{3} s^{7}+\frac{1}{3} s^{8}+\frac{1}{3} s^{9}$ & $c$ & $\frac{1}{3} s^{1}+\frac{1}{3} s^{4}+\frac{1}{3} s^{7}$ \\
\hline$s^{8}$ & $D$ & $\frac{1}{3} s^{7}+\frac{1}{3} s^{8}+\frac{1}{3} s^{9}$ & $d$ & $1_{s^{8}}$ \\
\hline$s^{9}$ & $D$ & $\frac{1}{3} s^{7}+\frac{1}{3} s^{8}+\frac{1}{3} s^{9}$ & $e$ & $1_{s^{9}}$ \\
\hline
\end{tabular}

Figure 2.2

This is an instance of the Preliminary Observation for Rationalizability. State $s^{1}$ is contained in the set $\left\{s^{1}, s^{2}, s^{4}, s^{5}\right\}$. At every state in this set both players are rational, the set of action profiles chosen at those states is $\{C, D\} \times\{c, d\}$ and it is mutual knowledge that the actions chosen is in $\{C, D\} \times\{c, d\}$. But it is not common knowledge that the actions chosen at $s^{1}$ are in $\{C, D\} \times\{c, d\}$. Though Ann knows that Bob chooses in $\{c, d\}$, she does not know that he knows this; indeed, she considers possible that he considers possible that she considers possible that he will play e. Moreover, though both players are rational at $s^{1}$, there is not even mutual 
knowledge of rationality there: at that state Bob considers possible that the true state is $s^{7}$, where Ann chooses $D$ with an expected payoff of $\frac{1}{3}$, rather than $C$, with an expected payoff of $\frac{4}{3}$; thus Ann is irrational at $s^{7}$.

This example also serves to illustrate the content of Theorem AB. At state $s^{2}$ the support of Ann's conjecture is $\{c, d\}$, the support of Bob's conjecture is $\{C, D\}$. It is also mutually known at $s^{2}$ that: (a) both players are rational, and (b) the support of Ann's conjecture is contaned in $\{c, d\}$ while the support of Bob's conjecture is contained in $\{\mathrm{C}, \mathrm{D}\}$. Indeed, these supports contain only rationalizable actions and, as the corollary to Theorem $\mathrm{AB}$ dictates, the actions chosen at $s^{2}$ are also rationalizable.

\subsection{Theorems, Proofs}

I now state and prove the main results of the paper formally.

Preliminary Observation for Rationalizability: Fix $g$ and $Z \subset$ A. Let $\widetilde{S}$ be a set of states where only-and-all the profiles in $Z$ are played, that is $Z=A(\widetilde{S})$. If at every state in $\widetilde{S}$ the actual game is $g$, everyone is rational and it is mutually known that $\mathbf{a} \in Z$ then $\times_{i \in N} Z_{i}$ is a set of action profiles with the best response property and the actions chosen at every state in $\widetilde{S}$ are rationalizable for $g$.

Proof. Need to show that any action $a_{i}$ in $Z_{i}$ maximizes the expectation of $g_{i}$ given a conjecture whose support is a subset of $Z_{-i}$. Pick $a_{i}$ in $Z_{i}$. Then there is a state $s$ in $\widetilde{S}$ such that $\mathbf{a}_{i}(s)=a_{i}$. At such state $s$ player $i$ maximizes his payoff 
function $g_{i}$ with respect to his conjecture $\phi^{i}(s)$. Since $[\mathbf{a} \in Z]$ is mutual knowledge at $s$ we have that $p\left([\mathbf{a} \in Z] ; s_{i}\right)=1$. Therefore, $\operatorname{supp} \phi^{i}(s) \subseteq Z_{-i}$. This shows that $\times_{i \in N} Z_{i}$ has the best response property. Now pick any state $s^{\prime}$ in $\widetilde{S}$. By definition, $\mathbf{a}\left(s^{\prime}\right) \in Z$ and hence the actions chosen at every state in $\widetilde{S}$ are rationalizable for $g$.

Theorem AB: Let $D:=\left(D^{i}\right)_{i \in N}$ with $D^{i} \subseteq A_{-i}$ be an $n$-tuple of supports of conjectures. Let $D_{i}^{j}$ be the projection of $D^{j}$ on $A_{i}$. Suppose that at some state $s$ supp $\phi(s)=D$, it is mutually known that payoff functions are $g$, that the players are rational, and that for every player $i$ supp $\phi_{i} \subseteq D^{i}$. Define the distributed conjecture about $i$ to be $C_{i}:=\cup_{j \neq i} D_{i}^{j}$. Then $\times_{i \in N} C_{i}$ is a set of action profiles with the best response property and, therefore, a set of rationalizable action profiles of $g$.

Proof. Need to show that any action $a_{i}$ in $C_{i}$ maximizes $g_{i}$ with respect to a conjecture whose support is a subset of $C_{-i}$. Pick $a_{i}$ in $C_{i}$ and hence in $D_{i}^{j}$ for some $j$. The proof is similar to the proof of Lemma 4.2 in Aumann and Brandenburger (1995). By assumption, supp $\phi^{j}(s)=D^{j}$. So $j$ attributes positive probability at $s$ to $\left[\mathbf{a}_{i}=a_{i}\right]$. Also, $j$ attributes probability 1 at $s$ to the following events: $i$ 's payoff function is $g_{i}, i$ is rational, and $i$ 's conjecture has support contained in $D^{i}$. So there is a state $t$ at which all four events obtain: $i$ 's action is $a_{i}, i$ 's payoff function is $g_{i}, i$ is rational, and $i$ 's conjecture has support contained in $D^{i}$. By definition, $D_{j}^{i} \subseteq C_{j}$, so $D^{i} \subseteq C_{-i}$ and $a_{i}$ maximizes $g_{i}$ with respect to a conjecture whose support is a subset 
of $C_{-i}$.

LEMMA L: If $\times_{i \in N} E_{i}$ is a set of profiles of strategies with the best response property and $a_{i}$ is a best response to a belief with support contained in $E_{-i}$, then $a_{i}$ is a rationalizable action.

Proof. If $a_{i}$ is in $E_{i}$ there is nothing to prove. If this is not the case consider the set $\times_{j \in N} E_{j}^{\prime}$ where $E_{i}^{\prime}=E_{i} \cup\left\{a_{i}\right\}$ and $E_{j}^{\prime}=E_{j}^{\prime}$ for $j \neq i$. To see that $\times_{j \in N} E_{j}^{\prime}$ has the best response property pick an action $a_{j}$ in $E_{j}^{\prime}$. If $a_{j}=a_{i}$ then, by hypothesis, $a_{j}$ is a best response to a belief with support contained in $E_{-j}$ and therefore in $E_{-j}^{\prime}$. If $a_{j} \neq a_{i}$ then $a_{j}$ is in $E_{j}$ and hence it is a best response to a belief with support contained in $E_{-j}$ and therefore in $E_{-j}^{\prime}$. This means that $a_{i}$ is a rationalizable action since, by construction, $a_{i}$ is in $E_{i}^{\prime}$.

Corollary 1 Under the same hypotheses of Theorem AB the actions chosen by the players at $s$ are rationalizable.

Proof. Under the hypotheses of Theorem $\mathrm{AB}$ the set $\times_{i \in N} C_{i}$ has the best response property. Since each player $i$ is rational at $s$ the action $\mathbf{a}_{i}(s)$ is a best response to a belief with support contained in $D^{i}$,which is, by construction, contained in $C_{-i}$. Hence, by Lemma L, action $\mathbf{a}_{i}(s)$ is rationalizable for every player $i$ in $N$. 


\section{Discussion}

a. The Conventional Wisdom. The purpose of this paper is not to argue that the conventional characterizations of rationalizability that rely heavily on common knowledge assumptions are wrong or inadequate; it is simply to point out that alternative characterizations, that do not require common knowledge of anything, are available. ${ }^{6}$ This is important to point out because the conventional wisdom leads us too quickly into thinking that there is no natural model of rationalizability other than the one based on common knowledge assumptions. ${ }^{7}$

\footnotetext{
${ }^{6}$ To wit: literature surveys (such as Dekel and Gul, 1997, and Battigalli and Bonanno, 1999) present only characterizations of rationalizability that depend on common knowledge assumptions. Therefore, to the extent that characterizations of rationalizability that do not require common knowledge assumptions were viewed as important by these authors, these results were largely unavailable at the time they wrote their surveys.

${ }^{7}$ For example, Brandenburger (1992) suggested that characterizations of Nash equilibrium that do not assume common knowledge of anything seem surprising because "common knowledge plays a role in [justifying correlated rationalizability]" (p.90). Binmore (1990), in turn, wrote: "To sustain the conclusion that nothing can be said about a game beyond the fact that a 'rationalizable' strategy will be played, one needs an explanation of how it comes about that the player's utilities are commonly known, but nothing whatever is known about their beliefs" (p. 4). Milgrom and Roberts (1991) wrote: "models of learning in games (...) where the players can use only information about past play are (...) in contrast with (...) approaches [such as] rationalizability [in that] they allow weight to be placed only on payoff information" (p. 85). Fundenberg and Tirole (1991) explain that "subjective correlated equilibrium is less restrictive than rationalizability [because it] allows each player's belief
} 
It is also not my purpose to suggest that the conditions that lead to rationalizability in the present paper are particularly weaker than the traditional conditions based on common knowledge assumptions. While the assumption of common knowledge is untenable in many situations, ${ }^{8}$ assumptions such as mutual knowledge of actions, payoffs, and conjectures are difficult to justify, too. Indeed, it is interesting to note that rationalizability can be characterized exactly by using an assumption about what the players know about the actions chosen by others because it was precisely a deep insatisfaction with this "rational expectations" assumption that led to the development of rationalizability. ${ }^{9}$ This fact suggests that the restrictions imposed by rationalizability, while weaker, are not unlike those imposed by Nash and are, therefore, "equilibriumlike." Brandenburger and Dekel (1987) reached a similar conclusion in a different setup.

b. Interpretation of the Preliminary Observation for Rationalizability. So far in the epistemic literature there have been two kinds of results. In the first kind of results, sometimes called "local" results, one makes assumptions about what is true about his opponents to be completely arbitrary, and thus cannot capture the restrictions implied by common knowledge of the payoffs" (p. 60).

${ }^{8}$ As seen, for example, in Fagin et. al. (1995).

${ }^{9}$ Despite the similarity between the assumptions it is clear that the assumption that players know that the actions chosen belong to some set Z (to which they truly belong to) is much weaker than assuming that they actually know the exact action profile chosen. 
regarding the player's knowledge, belief, rationality, etc. at a given state of the world, and then verifies what might be true, for example, regarding the actions chosen by the players, at that state. The second kind of results, sometimes called "global results," answer questions about what kind of inference an outside observer can make about the kinds of actions that are chosen across the entire state space based on what the observer knows to be true regarding the player's knowledge, belief, rationality, etc. at every state of the world. While Theorem $\mathrm{AB}$ is clearly a result of the first kind, the Preliminary Observation for Rationalizability appears to be of a third type altogether, as it neither fixes an individual state, nor takes an outside observer perspective. The Preliminary Observation appears to have features of both perspectives.

Indeed, I believe that to give an appropriate interpretation to the Preliminary Observation it is useful to adopt a third perspective: one in which there is an observer to the strategic interaction at hand distinct from the players of the game, but an observer whose knowledge and belief about the game is formalized the same way one formalizes the knowledge and the beliefs of the players. I call this the inside observer perspective. Here is a way to adapt this perspective to generate a "local" version of the Preliminary Observation for Rationalizability.

A model of $\mathcal{G}$ with observer is simply a model of $\mathcal{G}$ where each state also specifies beliefs for the inside observer, that is, a probability measure over the set $\times_{j \in N} S_{j}$.

Theorem IO: Fix $g$ and $Z \subset A$. Suppose that at some state $s$ the support of 
the inside observer's conjecture over the actions of the players is Z. Suppose further that the inside observer knows at $s$ that: payoff functions are $g$, that the players are rational, and that it is mutual knowledge among the players that $a \in Z$. Then the inside observer knows that a rationalizable action profile is chosen at $s$.

The proof of this result closely follows the proof of Theorem AB and I omit it here.

Notice that the knowledge of the inside observer is modelled in the same way as the knowledge of the players. Notice also that what the inside observer knows and what the players know need not coincide.

The inside observer perspective can be useful for generating results when it may be important to make precise the distinctions between what the participants in a social interaction know and believe, and what an observer different from the players (but part of their environment nevertheless) can know and believe. This can be of use as we, game theorists, are part of the very world we are trying to understand. Despite this, we often do not model ourselves as players in that world because there are typically weak strategic links between us and the strategic interactions we study. We do, however, share a similar cognitive representation of the world as the players in the social interactions we study and sometimes it may then be of value to formally represent what we know (be it the same or not as what the players know and believe), in the same language that we use to represent what the players know and believe. 
Adopting the inside observer perspective allows us precisely to do this and the present paper shows that this perspective may yield fresh insights into otherwise well studied game theoretic concepts.

c. Converses. While the epistemic conditions for rationalizability identified in this paper are not necessary, there is a sense in which the converses to the results presented in this paper hold: Given a game $g$ there is a model of $g$ such that the conditions are fulfilled. Choose a model with a state space $S$ identified with the largest set $Z$ of strategy profiles with the best response property where the payoffs are the same at every state, the beliefs at each state are such that players choices at that state are rational and have support in $Z$, and players' knowledge is derived from their beliefs at each state. In particular, every such set $Z$ has at least one strategy profile $z$ that consists of strategies that are not weakly dominated for either player. Have the belief of every player $i$ have full support on $Z_{-i}$ at the state $s$ corresponding to profile z. It may then be verified that the conditions of the Preliminary Observation for Rationalizability are met for $S$ and the conditions for Theorem AB are met at state $s$.

d. Independent rationalizability. The notion of rationalizability developed by Bernheim (1984) and Pearce (1984) did not allow for correlated conjectures, that is, for beliefs such that knowing about the actions chosen by some players would convey information about the actions to be taken by other players. One may naturally wonder 
whether characterizations of such notion of independent rationalizability exist along the lines of the ones developed in this paper for (correlated) rationalizability.

The answer to this question is a "qualified yes." Characterizations that do not assume common knowledge of anything do exist, although they do require an explicit assumption of the conjectures of the players being independent.

Preliminary Observation for Independent Rationalizability: In addition to the assumptions in the Preliminary Observation for Rationalizability, assume that at every state in $\widetilde{S}$ the conjecture of each player $i$ about each other player $j$ are independent of $i$ 's conjecture about all other players. Then $\times_{i \in N} Z_{i}$ is a set of independent rationalizable action profiles for $g$.

Theorem AB for Independent Rationalizability: In addition to the assumptions in Theorem AB, assume that it is mutual knowledge at s that the conjecture of each player $i$ about each other player $j$ are independent of $i$ 's conjecture about all other players. Then $\times_{i \in N} C_{i}$ is a set of independent rationalizable action profiles for $g$.

The explicit assumption of independence of conjectures make these result of somewhat limited interest in light of the subjectivist context in which the interactive epistemology literature is currently being developed. It is, nevertheless, important to recall that the standard characterization of independent rationalizability in terms of common knowledge assumptions does requires common knowledge of this indepen- 
dence (as well as common knowledge of rationality and payoffs). ${ }^{10}$ This means that both the Preliminary Observation and Theorem AB for independent rationalizability are still of interest relative to the well known characterization in terms of common knowledge.

A final remark on independent conjectures is in order. One may wonder whether assuming independence can be avoided altogether. I have investigated two routes to providing an answer to this question. First, there is the possibility that independence can be obtained along the lines of Theorem B in Aumann and Brandenburger (1995), who derive independent (and identical) conjectures out of assuming a common prior and common knowledge of conjectures. One may then conjecture that common knowledge of supports of the conjectures, or common knowledge of supports plus a common prior may lead to the conjectures to be independent (albeit not necessarily identical).

This turns out not to be the case. To see this consider a three player game where $A_{1}=\{C, D\}, A_{2}=\{L, R\}$ and $A_{3}=\{W, E\}$, with conjectures at each state induced by the common prior in Figure 3.1. Each type is denoted by the same letter as its action, and a subscript is added.

\footnotetext{
${ }^{10}$ See e.g., Tan and Werlang (1988).
} 


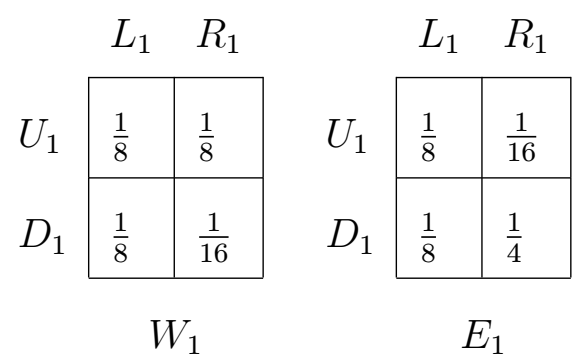

FiguRe 3.1

In this example the support of the conjecture of every player about the other two players is the same at every state of the world, hence the supports of the conjectures are common knowledge among the players. Conjectures themseves, however, are not common knowledge at any state; they are not even mutual knowledge at any state. And, in fact, conjectures are not independent at any state.

So nothing less than a common prior plus common knowledge of the conjectures themselves seems to be required for conjectures to be independent. But these assumptions, together with mutual knowledge of rationality and payoffs characterize Nash equilibrium, not independent rationalizability.

Another route to avoid assuming independence directly is to identify necessary and sufficient conditions for independence, such as the ones implied by the work of Geiger, Meek and Sturmfels (2006) for the study of graphical models and Bayesian networks. The conditions would entail assuming that the support of the conjectures 
have a product structure and verifying that the conjectures, for every player $i$, are such that

$$
\sum_{a_{-i} \in A_{-i}}\left(u_{a_{-i}}-v_{a_{-i}}\right) \log \phi_{i}\left(a_{-i} ; s_{i}\right)=0
$$

whenever

$$
\sum_{a_{k} \in A_{-\{i, j\}}}\left(u_{a_{j} a_{k}}-v_{a_{j} a_{k}}\right)=0 \text { for all } j \neq i, \text { all } a_{j} \in A_{j}
$$

and $u_{a_{-i}}$ and $v_{a_{-i}}$ are arbitrary integers. I find those conditions not easier to motivate that independence itself.

To conclude: it seems difficult to dispense with the independence assumptions in a characterization of independent rationalizability. Despite this, common knowledge of this independence, or rationality, or payoffs, are not necessary to characterize independent rationalizability as was previously believed.

e. Robustness of the Results. The results presented above are robust to weakening mutual knowledge to almost mutual 1 belief. ${ }^{11}$ To state this version of the results recall that an event $E$ is $q$-believed by player $i$ at $s$ when $p\left(E ; s_{i}\right) \geq q$ and almost 1 belief by player $i$ at $s$ if it is $q$-believed by player $i$ at $s$ for $q$ close to one. Now the results:

Robust Preliminary Observation for Rationalizability: Fix $g$ and $Z \subset A$. Let $\widetilde{S}$ be a set of states where only-and-all the profiles in $Z$ are played, that is

\footnotetext{
${ }^{11}$ These robustness results are inspired in Proposition 9 in Dekel and Gul (1997), a robustness check of whether common knowledge of rationality leads to rationalizability.
} 
$Z=A(\widetilde{S})$. Given a model of $g$ there is a $\bar{q} \in(0,1)$ such that if at every state in $\widetilde{S}$ the actual game is $g$, everyone is rational and it is mutual $q$-belief for $q>\bar{q}$ that $\mathbf{a} \in Z$, then $\times_{i \in N} Z_{i}$ is a set of action profiles with the best response property and the actions chosen at $\widetilde{S}$ are rationalizable for $g$.

Robust Theorem AB: Let $g$ be a game and $D:=\left(D^{i}\right)_{i \in N}$ with $D^{i} \subseteq A_{-i}$ be an $n$-tuple of supports of conjectures. Let $D_{i}^{j}$ be the projection of $D^{j}$ on $A_{i}$. Define the distributed conjecture about $i$ to be $C_{i}:=\cup_{j \neq i} D_{i}^{j}$.

Given a model of $g$ there is a $\bar{q} \in(0,1)$ such that if at some state supp $\phi(s)=D$, it is a mutual $q$-belief for $q>\bar{q}$ that payoff functions are $g$, that the players are rational, and that for every $i$ supp $\phi_{i} \subseteq D^{i}$, then $\times_{i \in N} C_{i}$ is a set of action profiles with the best response property and, therefore, a set of rationalizable action profiles of $g$.

The proofs of these results closely follow the proofs of the original results and I omit them here.

f. Weakly dominated strategies. Games without weakly dominated strategies allow for a simpler version of Theorem AB. It can be verified that all that is needed for a characterization of rationalizability at a given state is mutual knowledge of rationality, of payoffs, and of the support of the conjectures of the players. I omit the details here.

g. Learning, Evolution and Rationalizability. A basic result from the literature on learning in games is that adaptive learning leads to rationalizability (Milgrom and 
Roberts, 1991). ${ }^{12}$ Gul (1996), who independently developed results similar to those in Milgrom and Roberts (1991), interprets these results as providing examples of learning models where "rationality becomes common knowledge". This is, in general, viewed as surprising since adaptive learners know nothing about the rationality or the payoffs of others despite the strong link that exists between rationalizability and common knowledge of both rationality and payoffs. Results of this sort are often used to emphasize how the learning theoretic approach can "substitute for high levels of knowledge and deductive powers on the part of individuals"13 and therefore provide better foundations for rationalizability than the epistemic approach.

It is my belief that, in light of the results presented in the present paper, a different interpretation of the seminal results by Milgrom and Roberts (1991) and Gul (1996) is required. One can view these results as hinting at the existence of simpler epistemic conditions for rationalizability, that do not require common knowledge of anything. With the benefit of hindsight, they clearly point to the content of the Preliminary Observation for Rationalizability: after a sufficiently long time, adaptive learners play a near best reply to their beliefs, which are correctly specified in the sense that they

\footnotetext{
${ }^{12}$ Adaptive learning takes place when each player "eventually chooses only strategies that are nearly best replies to some probability distribution over his competitors' joint strategies, where near zero probability is assigned to strategies that have not been played for a sufficiently long time." (Milgrom and Roberts, 1991, p. 85).

${ }^{13}$ Young (1998, p. 112).
} 
place probability near one to strategies that arise from all players choosing, in fact, only near best replies to their beliefs. This says nothing, whatever, about whether players learn anything about the rationality of others, since these adaptive models are silent about what the players may know or learn about payoffs. Consequently, the interpretation of Proposition 9 in Gul (1996) (i.e., that rationality becomes common knowledge because only rationalizable strategies will eventually be played in the adaptive learning model) needs to be reexamined. ${ }^{14}$

h. Rationalizable Conjectural Equilibrium. Rubinstein and Wolinsky (1994) developed a solution concept intended to capture the steady state of recurring play of a game. Such solution concept, termed rationalizable conjectural equilibrium (RCE), corresponds loosely to strategy profiles such that (a) players best respond given their conjectures, (b) their conjectures are not contradicted by the profile chosen, and (c) conditions (a) and (b), together with the structure of the game and the monitoring technology, are common knowledge among the players. Interestingly, the set of RCE contains the set of Nash equilibria, and it is contained in the set of correlated rationalizable strategies.

Just as with the notion of rationalizability, RCE has been a concept intimately linked to the idea of the rationality of the players and of the structure of the game being commonly known. This intuition has been captured in Rubinstein and Wolinsky ${ }^{14}$ See also Young (1998, p. 112). 
(1994) and Battigalli (1999). Below I show that, just as with Nash Equilibrium and rationalizability, there are sparse conditions not involving common knowledge of anything that lead to RCE.

To do this I need to introduce some additional notation. A game for Rubinstein and Wolinsky (1994) specifies, apart from payoffs, strategies and players, signal functions $\mu_{i}: A \rightarrow T_{i}$ for each player $i$. The interpretation is that element $t_{i}=\mu_{i}(a)$ is the signal that player $i$ privately observes when all player choose the actions that make up the profile $a$. Profile $a^{*}$ is a rationalizable conjectural equilibrium if for each player $j$ there is a set $B_{j} \subseteq A_{j} \times T_{j}$ such that, for all $i,\left(a_{i}^{*}, \mu_{i}\left(a^{*}\right)\right) \in B_{i}$ and every $\left(a_{j}, t_{j}\right)$ in $B_{j}$ is such that $a_{j}$ is a best response to a belief $\alpha_{j}$ with support contained in

(i) $\left\{a_{-j}: \mu_{j}\left(a_{j}, a_{-j}\right)=t_{j}\right\}$, and

(ii) $\left\{a_{-j}: \forall k \neq j\left(a_{k}, \mu_{k}\left(a_{j}, a_{-j}\right)\right) \in B_{k}\right\}$.

In a RCE "each player's action is optimal, given a player's conjecture about what the other players' actions will be. The player's conjecture has to be consistent with the signal that he has on the actions the others intend to take, with the knowledge of his own action and with the knowledge that the other players follow similar reasoning." 15

In what follows a game form $\mathcal{G}^{\mu}$ is obtained from adding to game form $\mathcal{G}$ a signal function $\mu_{i}$ for each player $i$.

\footnotetext{
${ }^{15}$ Rubinstein and Wolinsky (1994, p. 302).
} 
A model of game form $\mathcal{G}^{\mu}$ is a model of $\mathcal{G}$ where each state $s$ also specifies the signal $\mu_{i}(\mathbf{a}(s))$ that each player $i$ receives at that state. As before, player $i$ 's conjecture at $s$ is $\phi^{i}(s)$. The conjecture of $i$ is confirmed at $s$ if player $i$ only puts positive probability at $s$ on actions for the opponents that would generate the signal received by player $i$ given player $i$ 's choice at $s$, namely, if every element in the support of $\phi^{i}(s)$ is contained in $\left\{a_{-i}: \mu_{i}\left(\mathbf{a}_{i}\left(s_{i}\right), a_{-i}\right)=\mu_{i}(\mathbf{a}(s))\right\}$.

Preliminary Observation for RCE: Fix $g$ and $Z \subset$ A. Let $\widetilde{S}$ be $a$ set of states where only-and-all the profiles in $Z$ are played, that is $Z=A(\widetilde{S})$. If at every state $s$ in $\widetilde{S}$ the actual game is g, everyone is rational, the conjectures of the players are confirmed and it is mutually known that $\mathbf{a} \in Z$. Then the action profile chosen at $s$ is a $R C E$ for $g$.

Proof. For each player $j$ let $B_{j}=\left\{\left(\mathbf{a}_{j}\left(s^{\prime}\right), \mu_{j}\left(\mathbf{a}\left(s^{\prime}\right)\right)\right): s^{\prime} \in S_{Z}\right\}$. Therefore, since $s$ is in $S,\left(\mathbf{a}_{i}(s), \mu_{i}(\mathbf{a}(s))\right) \in B_{i}$ for every player $i$. Now pick $\left(a_{j}, t_{j}\right)$ in $B_{j}$. By definition, action $a_{j}$ is chosen at some state $s^{\prime}$ in $S$ where $\mu_{j}\left(\mathbf{a}\left(s^{\prime}\right)\right)=t_{j}$. Consequently, $a_{j}$ is a best response to belief $\phi^{j}\left(s^{\prime}\right)$. Since the conjectures of the players are confirmed at $s^{\prime}$, the support of $\phi^{j}\left(s^{\prime}\right)$ is contained in $\left\{a_{-j}: \mu_{j}\left(a_{j}, a_{-j}\right)=t_{j}\right\}$. It remains to show that for all $a_{-j}$ in the support of $\phi^{j}\left(s^{\prime}\right)$ and for all $k \neq j$ it is the case that $\left(a_{k}, \mu_{k}\left(a_{j}, a_{-j}\right)\right) \in B_{k}$. Since $[\mathbf{a} \in Z]$ is mutual knowledge at $s^{\prime}$ we have that $p\left([\mathbf{a} \in Z] ; s_{i}^{\prime}\right)=1$. Consequently, for any $a_{-j}$ in supp $\phi^{j}\left(s^{\prime}\right)$ there is a state $s^{\prime \prime}$ in $\widetilde{S}$ such that $\mathbf{a}_{-j}\left(s^{\prime \prime}\right)=a_{-j}$ and $\mathbf{a}_{j}\left(s^{\prime \prime}\right)=\mathbf{a}_{j}\left(s^{\prime}\right)$. Since $s^{\prime \prime}$ is in $\widetilde{S}$, this means that for 
$k \neq j,\left(\mathbf{a}_{k}\left(s^{\prime \prime}\right), \mu_{k}\left(\mathbf{a}_{j}\left(s^{\prime}\right), \mathbf{a}_{-j}\left(s^{\prime \prime}\right)\right)\right) \in B_{k}$.

Theorem AB For RCE: Let $D:=\left(D^{i}\right)_{i \in N}$ with $D^{i} \subseteq A_{-i}$ be an $n$-tuple of supports of conjectures. Let $D_{i}^{j}$ be the projection of $D^{j}$ on $A_{i}$.

Suppose that at some state s supp $\phi(s)=D$, it is mutually known that payoff functions are $g$, that the players are rational, that the conjectures are confirmed and that for every $i$ supp $\phi_{i} \subseteq D^{i}$. Then the action profile chosen at $s$ is a RCE for $g$.

The proof of this result is very similar to the proofs constructed above and I omit it here.

i. Extensions. In this paper I have shown that there are epistemic conditions for rationalizability that do not assume common knowledge of anything, that these conditions are robust, and that they extend to refinements of rationalizability such as independent rationalizability and rationalizable conjectural equilibrium. These results lead us naturally to ask whether the same can be said about notions of rationalizability for games in extensive form (Pearce, 1984; Dekel, Fudenberg and Levine, 1999), and for games with incomplete information (Battigalli, 2003). These extensions are left for future work.

\section{References}

Armbruster, W., and W. Boege, "Bayesian Game Theory," in Game Theory and Related Topics, ed. by O. Moeschlin and D. Pallaschke. Amsterdam: North 
Holland, 1979.

Aumann, R., "Correlated Equilibrium as an Expression of Bayesian Rationality," Econometrica 55, 1-18, 1987.

Aumann, R., and A. Brandenburger, "Epistemic Conditions for Nash Equilibrium," Econometrica 63, 1161-1180, 1995.

Battigalli, P., "Rationalization and Incomplete Information," Advances in Theoretical Economics, 3(1), Article 3, 2003.

Battigalli, P., "A Comment on Non-Nash Equilibria, mimeo, 1999.

Battigalli, P. and G. Bonanno, "Recent Results on Belief, Knowledge and the Epistemic Foundations of Game Theory," Research in Economics 53, 149-225, 1999.

Bernheim, B. D., "Rationalizable Strategic Behavior," Econometrica 52, 1071028, 1984.

Bernheim, B. D., "Axiomatic Characterizations of Rational Choice in Strategic Environments," Scandinavian Journal of Economics 88, 473-488, 1986.

Brandenburger, A. and E. Dekel, "Rationalizability and Correlated Equilibria," Econometrica 55, 1391-1402, 1987. 
Dekel, E. and F. Gul, "Rationality and Knowledge in Game Theory," in D. Kreps and K. Wallis (eds.), Advances in Economics and Econometrics: Theory and Applications, Volume 1, Cambridge: Cambridge University Press, 1997.

Dekel, E., D. Fudenberg and D. Levine, "Payoff Information and Self-Confirming Equilibrium," Journal of Economic Theory 89, 165-185, 1999.

Fagin, R., J. Halpern, Y. Moses and M. Vardi, Reasoning About Knowledge, Cambridge: MIT Press, 1995.

Fudenberg, D. and J. Tirole, Game Theory, Cambridge: MIT Press, 1991.

Geanakoplos, J., "Common Knowledge," in R. Aumann and S. Hart (eds.), Handbook of Game Theory, Volume 2, Amsterdam: North-Holland, 1993.

Geiger, D., C. Meek, and B. Sturmfels, "On the Toric Algebra of Graphical Models," Annals of Statistics, forthcoming, 2006.

Gul, F. "Rationality and Coherent Theories of Strategic Behavior," Journal of Economic Theory 70, 1-29, 1996.

Harsanyi, J., "Games with Randomly Disturbed Payoffs: A New Rationale for Mixed action Equilibrium Points," International Journal of Game Theory 2, 1-23, 1973. 
Jordan, J., "Bayesian Learning in Games: A Non-Bayesian Perspective, in C. Biccieri, R. Jeffrey and B. Skyirms (eds.), The Dynamics of Norms, Cambridge University Press, 1996.

Milgrom, P. and J. Roberts, "Adaptive and Sophisticated Learning in Normal Form Games," Games and Economic Behavior 3, 82-100, 1991.

Nyarko, Y., "Bayesian Learning Leads to Correlated Equilibrium in Normal Form Games," Economic Theory 4, 821-841, 1994.

Nyarko, Y., "The Truth is in the Eye of the Beholder: or Equilibrium in Beliefs and Rational Learning in Games, manuscript, New York University, 1998.

Pearce, D., "Rationalizable Strategic Behavior and the Problem of Perfection," Econometrica 52, 1029-1050, 1984.

Tan, T. and S. Werlang, "The Bayesian Foundations of Solution Concepts of Games," Journal of Economic Theory 45, 370-391, 1988.

Young, P., Individual Strategy and Social Structure, Princeton: Princeton University Press, 1998. 